\title{
A Gephyrin-Related Mechanism Restraining Glycine Receptor Anchoring at GABAergic Synapses
}

\author{
Jochen Meier and Rosemarie Grantyn \\ Developmental Physiology, Johannes Müller Institute, Humboldt University Medical School (Charité), D-10117 Berlin, Germany
}

\begin{abstract}
Spinal cord neurons release glycine and GABA and accumulate glycine receptors (GlyRs) and $\mathrm{GABA}_{\mathrm{A}}$ receptors in the same postsynaptic densities. In contrast, supramedullar neurons prefer GABA as a neurotransmitter and exclude GlyRs from postsynaptic anchoring. The general aim of the present study was to elucidate the mechanisms underlying transmitter-appropriate receptor accumulation at inhibitory synapses. Specifically, we intended to clarify the molecular basis for the prohibition of GlyR accumulation in the postsynaptic densities of GABAergic synapses. A green fluorescent protein (GFP)-tagged gephyrin-binding loop of the GlyR $\beta$ subunit (GFP:: $\beta \mathrm{L}$ ) was used as a surrogate for full-length receptors to characterize the GlyR binding capacity of postsynaptic gephyrins in transfected neurons. Both in spinal cord neurons (SCNs) and hippocampal neurons (HNs) GFP:: $\beta$ L distribution displayed transmitter specificity; i.e., postsynaptic accumulation of GFP:: $\beta \mathrm{L}$ was high opposite terminals able to release glycine and low opposite purely GABAergic terminals. When comparing SCN and $\mathrm{HN}$ cultures we found that the level of mRNA coding for gephyrin splice variants containing the cassette C5 (C5-gephyrins) was significantly higher in HNs. In HNs depleted of C5-gephyrins, both GFP:: $\beta \mathrm{L}$ and endogenous GlyRs accumulated at postsynaptic GABAergic sites. Accordingly in SCNs, GFP-tagged C5-gephyrin displayed a preferential postsynaptic accumulation opposite GABAergic synapses. Comparison of glycinergic, mixed, and GABAergic synapses in SCNs showed that the degree of GlyR accumulation was inversely related to the amount of postsynaptic $\mathrm{C} 5$-gephyrin. These results identify the $\mathrm{C} 5$ splice variant of gephyrin as a factor regulating the transmitter-appropriate degree of GlyR accumulation at inhibitory synapses.
\end{abstract}

Key words: gephyrin; splicing; glycine receptor; transfection; spinal cord; hippocampus; anchoring

\section{Introduction}

Glycine and GABA are the two major inhibitory neurotransmitters in the CNS. They share the vesicular inhibitory amino acid transporter (VIAAT) (McIntire et al., 1997; Sagné et al., 1997; Gasnier, 2000) but use distinct plasma membrane transporters. Glycine transport into presynaptic terminals is performed by the neuronal glycine transporter isoform GlyT2 (Zafra et al., 1995a), whereas GABA transport is mediated by the neuronal GABA transporter (Radian et al., 1990). Presynaptic GABA levels also depend on the activity of the GABA-synthesizing enzyme glutamic acid decarboxylase (GAD) (Soghomonian and Martin, 1998). The presence of GlyT2 or GAD, or both, is taken as a criterion to assign a transmitter phenotype to presynaptic terminals. In spinal cord neurons ( $\mathrm{SCNs}$ ), inhibitory terminals release either glycine or GABA, or both transmitters (Jonas et al., 1998). Rostral to the medulla, the predominant neurotransmitter is GABA (Luhmann and Prince, 1991; Beaulieu et al., 1994; Zafra et al., 1995a).

As a rule, postsynaptic accumulation of neurotransmitter re-

Received June 19, 2003; revised Dec. 10, 2003; accepted Dec. 10, 2003.

This work was supported by the Deutsche Forschungsgemeinschaft (Grant SFB 515 B2 to R.G.). We express our gratitude to our colleagues H. Betz and A. Rodriguez-Tebar for valuable suggestions and critical reading of this manuscript. We thank U. Neumann for excellent technical assistance.

Correspondence should be addressed to Dr. Jochen Meier, Developmental Physiology, Johannes Müller Institute Humboldt University Medical School (Charité), Tucholskystrasse 2, D-10117 Berlin, Germany. E-mail: jochen.meier@charite.de.

DOI:10.1523/JNEUROSCI.4260-03.2004

Copyright $\odot 2004$ Society for Neuroscience $\quad$ 0270-6474/04/241398-08\$15.00/0 ceptors closely matches the transmitter phenotype of presynaptic terminals. A long-standing question is how this alignment between presynaptic and postsynaptic sites is achieved (Nicoll and Malenka, 1998). One way to address this question is to analyze postsynaptic changes associated with a developmental switch from one inhibitory neurotransmitter to another (Meier et al., 2002). In superior colliculus cultures, inhibitory synapses initially release glycine; later on they release GABA. At either developmental stage, postsynaptic receptor accumulation of the appropriate receptor matched the transmitter phenotype, and this coincided with an upregulation of a particular gephyrin isoform.

Gephyrin is a glycine receptor (GlyR)-tubulin-bridging molecule, initially purified in association with the GlyR $\beta$ subunit. It is composed of two domains that are homologous to the bacterial MogA and MoeA proteins (see Fig. 1A). A high degree of gephyrin diversity (see Fig. $1 B$ ) is generated by insertion of cassettes C1-C6 (Meier et al., 2000a; David-Watine, 2001; Reiss and Johnson, 2003). The resulting functional diversity of gephyrins has recently attracted considerable attention (Butler et al., 2000; Reiss et al., 2001; Rees et al., 2003). In developing collicular cultures, the disappearance of GlyRs from inhibitory synapses was associated with increased expression of gephyrin isoforms containing the cassette C5 (C5-gephyrins). Glutathione $S$-transferase pulldown assays showed that C5-gephyrins failed to interact with the gephyrin-binding loop of the GlyR $\beta$ subunit (Meier et al., 2000a); however, the MogA-homologous domain, where C5 is inserted, may not be involved directly in GlyR $\beta$ subunit binding 
(Sola et al., 2001). This is consistent with the location of the GlyR $\beta$ subunit binding domain within the C-terminal part (see Fig. $1 A$ ) of gephyrin (Rees et al., 2003). The MogA-homologous domain triggers trimerization, whereas the MoeA-homologous region mediates assembly into dimers (Xiang et al., 2001). Together, the MogA- and MoeA-homologous domains orchestrate the assembly of a highly ordered hexagonal protein lattice structure (Xiang et al., 2001).

Depending on the actual pattern of gephyrin isoforms in the postsynaptic neurons, GlyR stabilization might be either promoted or hindered; however, the possibility of a dual regulation of receptor access to postsynaptic densities has not yet received much consideration. Therefore the aim of the present study was (1) to further clarify the function of C5-gephyrins at inhibitory synapses and (2) to test the hypothesis that, depending on its molecular structure, postsynaptic gephyrins may either facilitate or suppress GlyR binding and in this way contribute to transmitter-appropriate GlyR localization.

\section{Materials and Methods}

cDNA constructs. Total RNA and cDNA were obtained as described (Meier et al., 2002). For the green fluorescent protein (GFP)-tagged gephyrin-binding loop of the GlyR $\beta$ subunit construct (GFP:: $\beta \mathrm{L}$ ), PCR amplimers corresponding to the gephyrin-binding loop of the GlyR $\beta$ subunit $(\beta \mathrm{L})$ were prepared from rat spinal cord cDNA using oligonucleotides $\left(5^{\prime}-3^{\prime}\right)$ GGGAGATCTAAAAGGGTTGAAGCTGAG and GGGGAATTCTCATCTTGCATAGAGGTCAATGC. After subcloning (using BglII and EcoRI) into the plasmids (Clontech, Palo Alto, CA) pEGFP-C1 and pDsRed2-C1, respectively, the final constructs were verified by DNA sequencing. The GFP-tagged C2- and C6-containing gephyrin isoform $(\mathrm{GeC} 2,6)$ was isolated by RT-PCR from total RNA of adult rat spinal cord. The absence of other cassettes was secured by PCR following a previously described protocol (Meier et al., 2002). To obtain the C2-, C5-, and C6-containing gephyrin clone $(\mathrm{GeC} 2,5,6)$, the cassette C5 was inserted into the $\mathrm{GeC} 2,6$ clone by site-directed mutagenesis (GeneEditor, Promega, Madison, WI) using the 5' phosphorylated oligonucleotide (5'-3') CGAGATGTCACACCAGAGAAATTCCCAACATTCCCATTTTGTGGGCTCCAGAAAGGGGCCACAAAAGAAGTAATAGAAC. Both cDNAs were finally subcloned into pEGFP-N1 vector (Clontech). Final constructs were subjected to DNA sequencing.

Cell culture and transfection. SCNs from embryonic day (E) 14 Wistar rats or hippocampal neurons (HNs) from E18 rats were prepared as described previously (Rao et al., 1998; Meier et al., 2000b) and maintained in B27- and 1\% FCS-supplemented Neurobasal medium (Brewer and Cotman, 1989). After neuron attachment, the coverslips were transferred (cell-side down) to dishes containing a confluent layer of spinal cord or hippocampal glial cells, respectively.

Transfection was performed after $8 \mathrm{~d}$ (HNs) or $12 \mathrm{~d}$ (SCNs) in vitro (DIV). Coverslips were transferred to wells containing the transfection medium (Neurobasal supplemented with $0.25 \mathrm{~mm}$ glutamine and $1 \%$ FCS) and incubated with complexes formed between $5 \mu$ l of Effectene transfection reagent (Qiagen, Hilden, Germany) and 300 ng of DNA. The manufacturer's transfection protocol was followed, except that the incubation time was reduced to 1.5 and $3 \mathrm{hr}$ for SCNs and HNs, respectively. This protocol ensured moderate expression levels of the transfected proteins. The GFP fluorescence was clearly visible $14-16 \mathrm{hr}$ after the transfection, and the cultures were transferred into a fixative and processed for immunocytochemistry.

Antisense experiments. To manipulate the expression of C5-gephyrins, we used the antisense phosphorothioate oligonucleotide shown in Figure 5 . We deliberately selected an overlapping region between the $3^{\prime}$ end of $\mathrm{C} 5$ and the downstream gephyrin cDNA sequence because the C5-cDNA sequence is not unique for gephyrin: a C5-cDNA sequence has also been found within an mRNA sequence (C57BL/6J mouse, chromosome 12, LOC217680, accession: XM_126999; the National Center for Biotechnology Information Annotation Project) coding for a potential mouse molybdopterin binding domain. Corresponding C5 sense oligonucleo- tides were designed according to the complementary cDNA sequence. Oligonucleotides were tetramethylrhodamine-5-(and-6-)isothiocyanate(TRITC) labeled at $5^{\prime}$, ensuring that only sense or antisense oligonucleotide-containing neurons were visualized. Three concentrations $(5,50$, and $500 \mathrm{~nm})$ of sense or antisense oligonucleotides were applied to co-transfect neurons together with GFP:: $\beta$ L. Finally, the effect of C5 antisense co-transfection on C5-mRNA depletion was verified by RT-PCR.

Antibodies. The monoclonal antibodies (mAbs) used in this study recognized gephyrin [mAb7a, 1:200 (Pfeiffer et al., 1984); Alexis Biochemicals, San Diego, CA], GAD65 [1:100 (Gottlieb et al., 1986); Chemicon, Temecula, CA] , and GlyRs [mAb2b, 1:50, mAb4a, 1:50; Alexis Biochemicals]. The polyclonal antibodies (pAbs) were guinea pig anti-VIAAT [1:800 (Dumoulin et al., 1999); Chemicon], sheep anti-GlyT2 [1:200 (Zafra et al., 1995a); Acris GmbH, Hiddenhausen, Germany], and rabbit anti-GAD65/67 [1:500, Chemicon]. For double- and triple-labeling experiments, $\mathrm{mAbs}$ and $\mathrm{pAbs}$ were always combined. Secondary antibodies were carboxymethyl indocyanine, fluorescein isothiocyanate, or Alexa Fluor 350 coupled and purchased from Jackson ImmunoResearch Laboratories (West Grove, PA), and Molecular Probes (Eugene, OR), respectively.

Immunocytochemistry and quantification. Immunofluorescence was performed as described previously (Meier et al., 2002). Images were acquired using a standard epifluorescence microscope (objective 100×; Axiovert, Carl Zeiss, Oberkochen, Germany), a 12-bit cooled CCD camera (Ch250, Photometrics, Tucson, AZ), and the software PMIS 4.1.4 (Remington, Munich, Germany). Appropriate filters (XF22, XF32, XF110-2, XF136-2; Omega Optical Inc., Brattleboro, VT) allowed the detection and separation of fluorescent signals. To show that the labeling was caused specifically by the primary antibody, we replaced it with similarly diluted normal serum from the same species. To distinguish between clusters and diffuse staining, the SD of the diffuse fluorescence signal was determined. Classification of a spot as cluster required that the fluorescence intensity exceeded three times the SD of the diffuse signal ( $3 \sigma$ criterion). For determination of surface areas and fluorescence intensities, NIH image software was used according to the procedure described previously (Meier et al., 2003). For determination of colocalization, images were merged. Images were scaled linearly, and colocalized spots were counted manually to avoid any bias introduced by electronic thresholding procedures. Colocalization indices were defined on the basis of spatial closeness between two or more fluorescent spots and expressed as the percentage fraction of the number of colocalized spots and the total number of a given immunoreactivity. For colocalization of two clusters of immunogens, we required that $>50 \%$ of the pixels reflecting one immunogen should show colocalization with a coherent cluster of the corresponding other immunogen. The region of interest comprised the soma and $50 \mu \mathrm{m}$ of proximal dendrites. Values are expressed as mean \pm SEM obtained from 15-20 cells in three independent experiments.

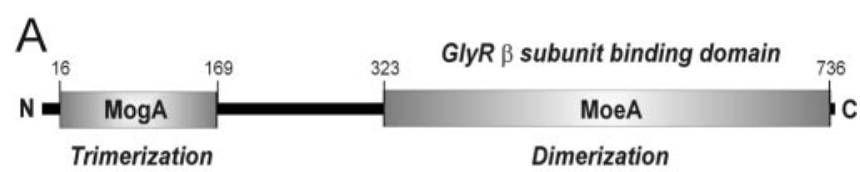

B

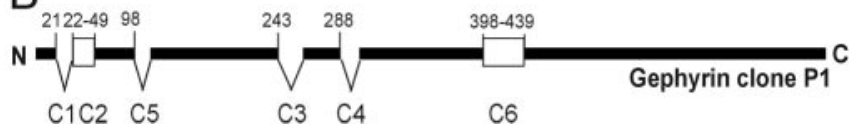

Figure 1. Gephyrin structure. $A$, Gephyrin is composed of two domains that are homologous to the bacterial proteins MogA and MoeA and separated by a linker region. The N-terminal domain is involved in trimer formation, whereas the C-terminal domain mediates dimerization and is required for GlyR binding (Rees et al., 2003). Together, both domains are presumably involved in the assembly of an ordered hexagonal lattice. $B$, The initially identified clone P1 (Prior et al., 1992) bearing cassettes C 2 and C6. Insertion of at least six distinct cassettes generates a large number of alternatively spliced gephyrin isoforms. Numbering identifies amino acid positions within the gephyrin open reading frame. $N$ and $C$ termini are indicated. 
Semiquantitative RT-PCR. RT-PCR was performed as described (Meier et al., 2002). Titration was performed to ensure that PCR reactions were performed in the linear range of amplification. The resulting number of cycles was 30 . To amplify GlyR subunits and gephyrin splice variants, oligonucleotides were designed as described previously (Meier et al., 2002). Rat $\beta$-actin $\left(5^{\prime}-3^{\prime}\right)$ TTGTAACAAACTGGGACGATATGG and GATCTTGATCTTCAT GGTGCTAG was always co-amplified in the same PCR reaction tube and served as a reference for the quantification of the total cDNA amount. For each test, three separate PCR experiments were performed on three separate RNA preparations. PCR products were separated using agarose gel electrophoresis and visualized by ethidium bromide staining. The intensity and volume of the analyzed PCR products were quantified using ImageQuant software (Molecular Dynamics, Sunnyvale, CA) and expressed as mean pixel intensity. Then a ratio was calculated between $\mathrm{C} 2+\mathrm{C} 5$ and $\mathrm{C} 2$, and between $\mathrm{C} 3$ and without $\mathrm{C} 3$ (w/o) bands, respectively. A ratio value of 1 indicates equal amounts of the compared PCR products. Molecular weight DNA marker (100 bp DNA ladder) was purchased from Invitrogen (Bethesda, MD).

\section{Results}

GFP:: $\beta \mathrm{L}$ accumulates at gephyrincontaining inhibitory postsynaptic sites The $\beta$ subunit is involved in GlyR binding to gephyrin (Fig. 1) and in its stabilization at inhibitory synapses (Meyer et al., 1995, 2001; Kneussel et al., 1999). Previous experiments have shown that the gephyrin-binding loop of the GlyR $\beta$ subunit suffices to report on gephyrin binding in vitro (Meier et al., 2000a). Here we used a GFP-tagged M3-M4 loop of the GlyR $\beta$ subunit as a surrogate for full-length, $\beta$ subunit-containing GlyRs to study the interaction with gephyrin in transfected neurons.

First, the in vitro binding capacity of GlyR $\beta \mathrm{L}$ to gephyrin was determined according to the procedure described by Kins et al (1999). African green monkey kidney (COS-7) cells were transfected with DsRed2-tagged GlyR $\beta \mathrm{L}$ (DsRed2:: $\beta \mathrm{L}$ ) and GFP-tagged C2- and C6-containing gephyrin [GeC2,6::GFP; corresponds to the $\mathrm{P} 1$ clone isolated by Prior et al. (1992)]. When coexpressed, DsRed2:: $\beta \mathrm{L}$ is recruited to the large somatic gephyrin C2,6::GFP aggregates in transfected COS-7 cells (Fig. $2 A, B$ ).

To determine whether GFP:: $\beta \mathrm{L}$ displays postsynaptic accumulation at inhibitory sites, we studied its distribution in SCNs. GFP:: $\beta \mathrm{L}$ aggregates became visible in the somatodendritic area $16 \mathrm{hr}$ after transfection (Fig. 2C). VIAAT immunofluorescence revealed that these aggregates were colocalized with $66.9 \pm 2.9 \%$ of inhibitory terminals (Fig. 2D,F). Triple im-
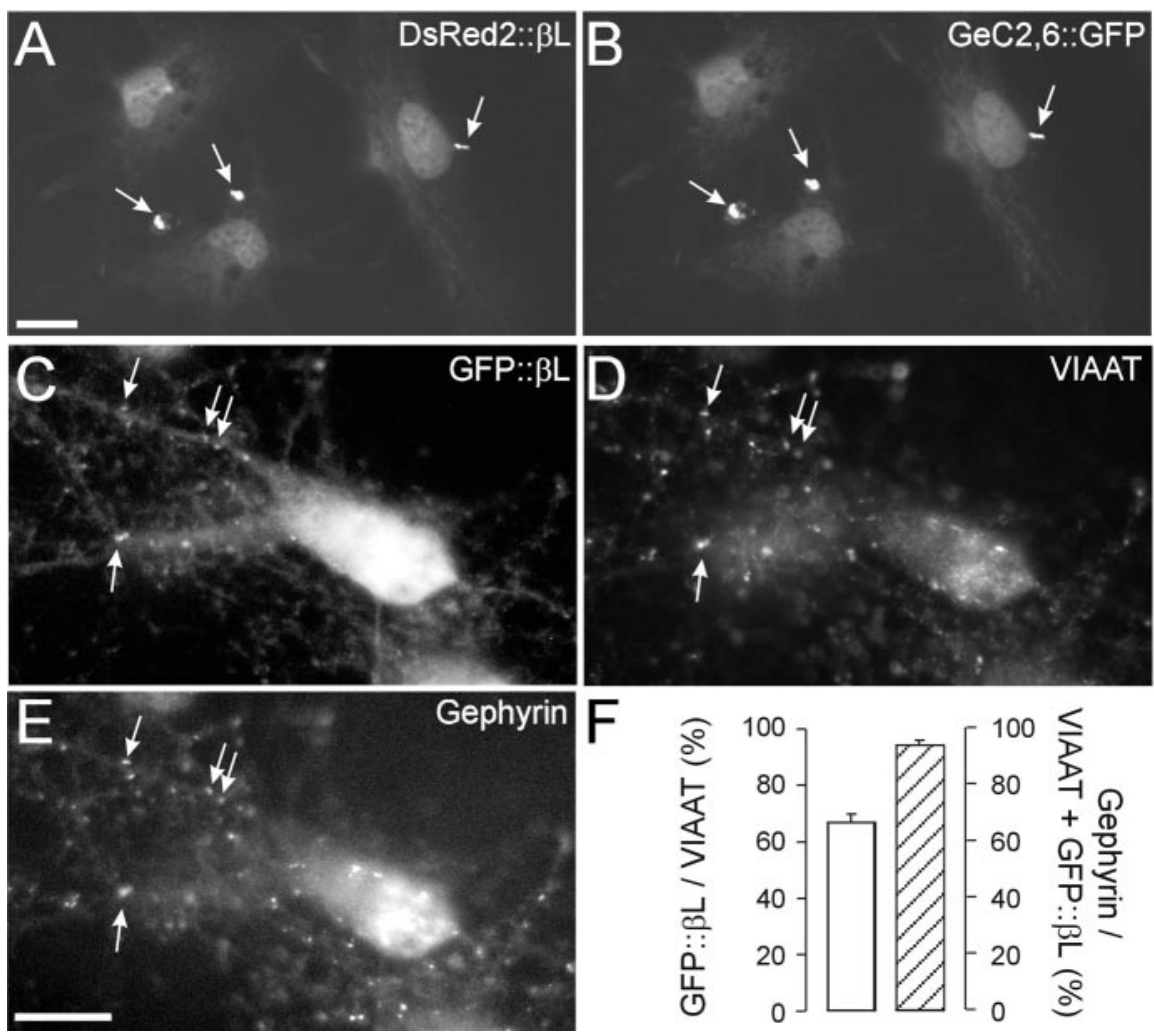

Figure 2. Accumulation of transfected GFP:: $\beta \mathrm{L}$ opposite inhibitory synaptic terminals in DIV12 SCNs. $A, B$, COS-7 cells transfected with DsRed2:: $\beta \mathrm{L}(A)$ and GeC2,6::GFP $(B)$. DsRed2:: $\beta$ L is trapped by intracellular gephyrin aggregates and therefore can be regarded as capable of binding to gephyrin. $C$, Image of a neuron containing GFP:: $\beta$ L. $D, E$, Corresponding endogenous VIAAT (D) and gephyrin $(E)$ immunoreactivity. F, Fraction of VIAAT-immunoreactive terminals that also contained GFP.: $\beta$ L (left $y$-axis) and fraction of VIAAT- and GFP:: $\beta$ L-immunoreactive synapses that also contained gephyrin (right $y$-axis). Note the high degree of colocalization between GFP:: $\beta \mathrm{L}$, VIAAT, and gephyrin. Scale bars, $10 \mu \mathrm{m}$.
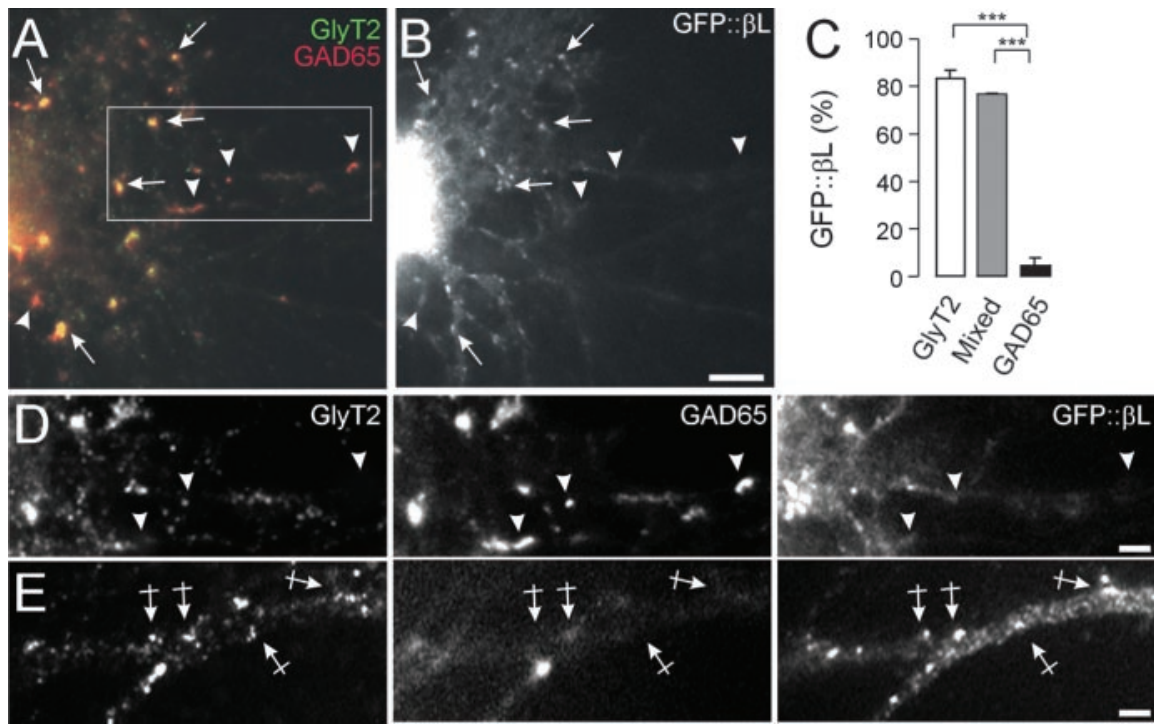

Figure 3. Transmitter-appropriate postsynaptic accumulation of transfected GFP:: $\beta \mathrm{L}$ in DIV12 SCNs. $A, B$, Same view field GlyT2 and GAD65 immunoreactivity of terminals $(A)$ making contact with a GFP:: $\beta$ L expressing neuron $(B)$. $C$, Fraction of glycinergic (GlyT2 + only), mixed (GlyT2 + and GAD65 +), or GABAergic (GAD65 + only) terminals facing GFP:: $\beta$ L clusters. $D$, Highpower views of the region delineated in $A$. Arrowheads show lack of GFP:: $\beta$ L accumulation opposite GAD65-only synapses. $E$, High-power views of a dendrite taken from another transfected neuron illustrating accumulation of GFP.: $\beta$ L opposite GlyT2-only synapses (crossed arrows). Note that GFP:: $\beta$ L colocalizes with glycinergic or mixed terminals (arrows, crossed arrows) but not with purely GABAergic terminals (arrowheads). Scale bars: $A, B, 5 \mu \mathrm{m} ; D, E, 2 \mu \mathrm{m}$. 

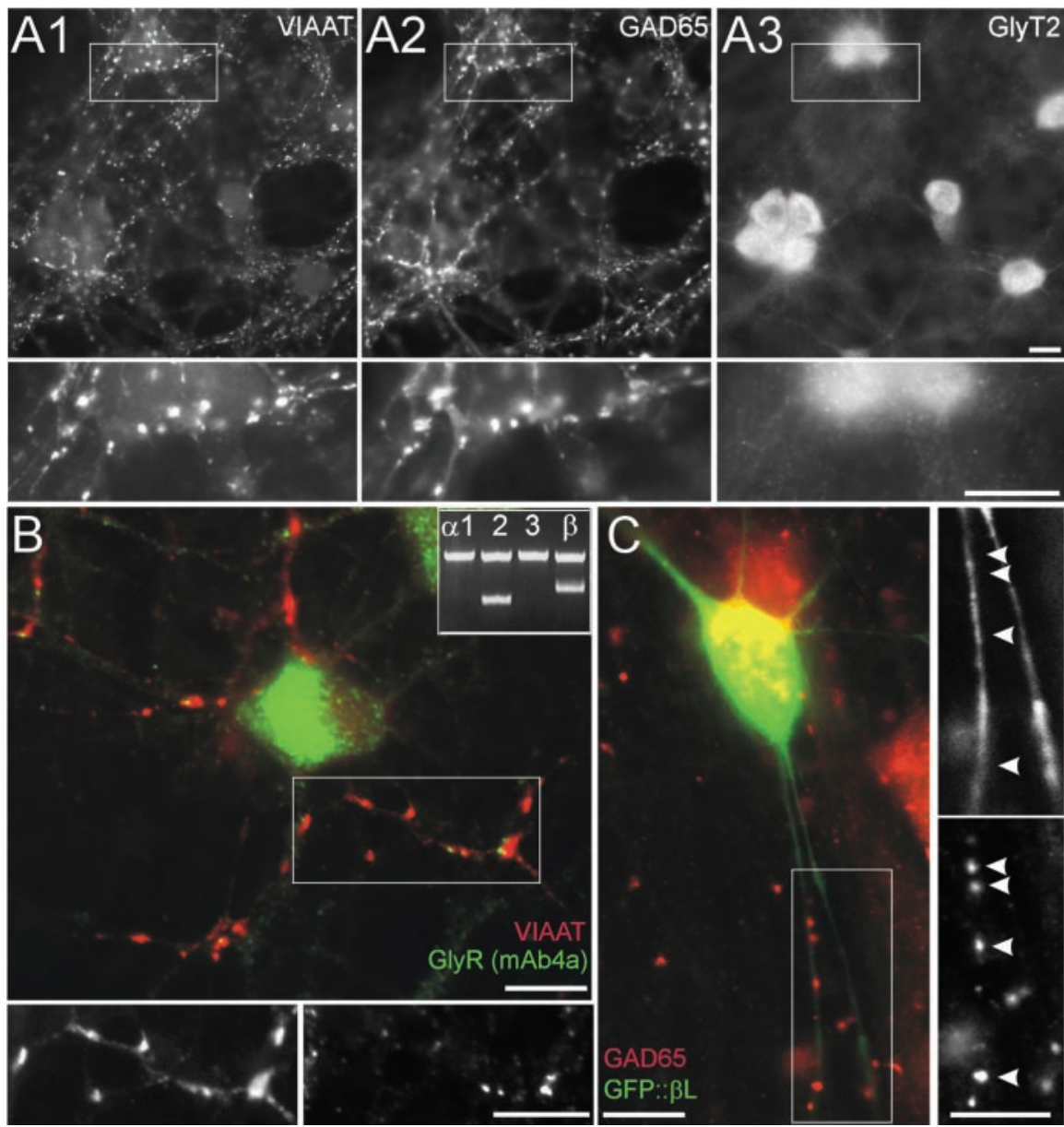

Figure 4. Lack of postsynaptic GFP:: $\beta$ L accumulation in DIV8 HNs. A, Triple immunofluorescence of VIAAT (A1), GAD65 (A2), and GlyT2 (A3). Note lack of GlyT2 immunoreactivity. B, Example of an HN expressing endogenous GlyRs (mAb4a) that were partially colocalized with VIAAT-containing terminals. Inset in $B$ shows the presence of mRNAs coding for GlyR $\alpha 2$ and $\beta$ subunits. Top bands in each lane represent $\beta$-actin amplification products. C, Distribution of transfected GFP:: $\beta$ L. Note the diffuse cytosolic localization of GFP:: $\beta$ L and its absence opposite GAD65-labeled terminals (arrowheads). Scale bars, $10 \mu \mathrm{m}$.

munofluorescence labeling revealed the close association of postsynaptic GFP:: $\beta \mathrm{L}$ with endogenous gephyrin $(94.9 \pm 1.8 \%)$ (Fig. $2 E, F)$. These results show that our GlyR $\beta \mathrm{L}$ construct is suitable to bind gephyrin and to accumulate at gephyrincontaining inhibitory sites in transfected SCNs.

\section{Transmitter-appropriate postsynaptic accumulation of} GFP:: $\beta \mathrm{L}$ in spinal cord neurons

SCNs were transfected with GFP:: $\beta \mathrm{L}$, and the transmitter phenotype of presynaptic terminals was determined by GlyT2 and GAD65 immunofluorescence (Fig. 3). GFP:: $\beta$ L accumulated opposite mixed and purely glycinergic terminals (Fig. $3 A, B$, arrows, $E$, crossed arrows, respectively). In contrast, postsynaptic sites opposite purely GABAergic terminals were mostly free of GFP:: $\beta \mathrm{L}$ (Fig. $3 A, B, D$, arrowheads). In detail, $85.0 \pm 3.8,77.7 \pm$ 0.6 , and $4.6 \pm 3.1 \%$ of glycinergic, mixed, and GABAergic synapses, respectively, contained GFP:: $\beta \mathrm{L}$ (Fig. $3 C$ ). To rule out the possibility that our experimental procedures interfered with inhibitory synaptogenesis, we determined the fraction of synapses with purely glycinergic, mixed, and purely GABAergic terminals. SCNs carried $15.4 \pm 2.5$ VIAAT-immunoreactive terminals per transfected neuron and view field. In agreement with previous results (Dumoulin et al., 2000), it was found that $25.9 \pm 0.2 \%$ of the terminals were glycinergic, $54.0 \pm 1.0 \%$ were mixed, and

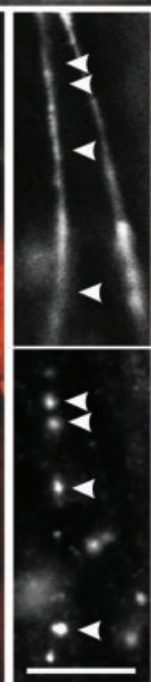

$20.1 \pm 1.2 \%$ were GABAergic. This indicates that formation and maintenance of inhibitory synapses were not seriously altered. We conclude that the distribution of GFP:: $\beta$ L fully reproduces the transmitterspecific pattern known from previous studies on endogenous full-length GlyRs.

\section{Diffuse intracellular distribution of GFP:: $\beta \mathrm{L}$ in hippocampal neurons}

In contrast to SCNs, inhibitory synapses between HNs were formed exclusively by GAD65-immunoreactive terminals (Fig. $4 A)$. In line with previous studies on $\mathrm{HNs}$ (Zafra et al., 1995a,b; Mori et al., 2002), all inhibitory synapses (Fig. 4A1, VIAAT) contained immunoreactivity for GAD65 (Fig. 4A2) but were free of GlyT2 (Fig. $4 A 3$ ). Therefore, VIAAT labeling was considered to identify GABAergic terminals in HNs. Nonetheless, HNs are known to express GlyRs (Ito and Cherubini, 1991), including functional heteromeric GlyRs composed of $\alpha 2$ and $\beta$ subunits (Mori et al., 2002; Thio et al., 2003), but in contrast to SCNs, the GlyRs of HNs are reported to localize in the nonsynaptic plasma membrane (Mori et al., 2002). RT-PCR experiments were performed to verify that under the given culture conditions HNs indeed expressed GlyR $\alpha 2$ and $\beta$; this was the case (Fig. $4 B$, inset). Immunostaining of the hippocampal cultures (Fig. $4 B$ ) revealed, in addition, that $33.4 \pm 3.4 \%$ of the GABAergic synapses contained clusters formed by endogenous GlyRs.

To determine whether these postsynaptic GlyR clusters require binding of the $\beta$ subunit to gephyrin, HNs were transfected with GFP:: $\beta \mathrm{L}$ (Fig. 4C). Considering that (1) GFP:: $\beta \mathrm{L}$ behaves like a soluble protein and (2) GFP:: $\beta$ L binding to gephyrin is prohibited in HNs, one should expect a diffuse cytosolic GFP:: $\beta$ L localization. Potential clusters were distinguished from diffuse distribution by the $3 \sigma$ criterion (see Materials and Methods). The distribution of GFP:: $\beta \mathrm{L}$ was indeed diffuse within the cytosol. No colocalization was found with GAD65-containing terminals (Fig. 4C, arrowheads). Thus, although endogenous GlyR clusters did occur at some of the GABAergic inhibitory terminals, binding of the GlyR $\beta$ subunit to the postsynaptic gephyrin scaffold was suppressed to the extent that overexpressed GFP:: $\beta \mathrm{L}$ assumed a diffuse distribution. HNs are therefore well suited to address the question of whether C5-gephyrins are responsible for keeping heteromeric $\beta$ subunit-containing GlyRs out of inhibitory synapses.

Hippocampal neurons differ from spinal cord neurons in the expression of C3- and C5-containing gephyrin splice variants To identify the mechanism that might serve to prohibit a postsynaptic stabilization of $\beta$ subunit-containing GlyRs, we analyzed HNs with RT-PCR (Fig. 5A-D). As compared with SCNs, HNs expressed C5-gephyrins at much higher levels (Fig. 5A,B). The ratio of $\mathrm{C} 2+\mathrm{C} 5$-gephyrin $\mathrm{cDNAs}$ versus $\mathrm{C} 2$-gephyrins without $\mathrm{C} 5(\mathrm{C} 2+\mathrm{C} 5 / \mathrm{C} 2)$ was significantly larger in HNs (Fig. $5 \mathrm{C})$. HNs 
also displayed a higher ratio of gephyrin cDNAs with cassette C3 (Fig. 5D). Because cassette $\mathrm{C} 3$ apparently does not alter binding of the GlyR $\beta$ subunit to gephyrin (Meier et al., 2000a), the results are in line with the idea that C5-gephyrins may indeed be responsible for the prohibition of heteromeric GlyR anchoring in postsynaptic GABAergic densities.

\section{C5-gephyrins are responsible for the exclusion of GFP:: $\beta \mathrm{L}$ from GABAergic synapses}

To verify the key role of C5-gephyrins in prohibiting GlyR binding to postsynaptic GABAergic domains, C5-gephyrin antisense experiments were designed as shown in Figure $5 E$ (details in Materials and Methods). Figure 5, $F$ and $G$, illustrates the C5 antisense oligonucleotide dosedependent reduction of C5-gephyrin mRNA levels in HNs. Note that the overall amount of C2-containing transcripts remained roughly unchanged: C5 antisensetreated neurons displayed a slight increase by 5 and $2 \%$ (at 50 and $500 \mathrm{~nm}$, respectively) in the total amount of $\mathrm{C} 2$-gephyrins (comparison of the combined intensity signal from the top plus bottom band at 0 , 50 , and $500 \mathrm{nM})$. We therefore concluded that C5 antisense treatment effectively downregulated the C5-containing gephyrin mRNA fraction. Accordingly, the C3containing gephyrin mRNA fraction was upregulated (Fig. $5 F, G$, bottom). This upregulation of $\mathrm{C} 3$-gephyrins most probably reflects the lack of cassette C3 in C5containing gephyrin isoforms.

$\mathrm{HNs}$ were then co-transfected with C5 antisense oligonucleotides and GFP:: $\beta \mathrm{L}$ to study the dependency of postsynaptic GFP:: $\beta \mathrm{L}$ accumulation on C5-gephyrins. C5-gephyrin-deficient HNs contained GFP:: $\beta \mathrm{L}$ aggregates opposite GABAergic terminals (Fig. 6A, arrows). The effect of $\mathrm{C} 5$ antisense oligonucleotide treatment on postsynaptic GFP:: $\beta \mathrm{L}$ accumulation was dose dependent (Fig. $6 \mathrm{~B}$ ); however, as indicated by an increase in the number of pyknotic nuclei (Hoechst stain; data not shown), transfection of $500 \mathrm{~nm} \mathrm{C5}$ antisense was not well tolerated by HNs. Whenever postsynaptic GFP:: $\beta \mathrm{L}$ aggregates were found in $\mathrm{C} 5$ antisense-transfected $\mathrm{HNs}$, they also colocalized with endogenous gephyrin $(96.6 \pm 1.8 \%)$ (Fig. 6C). In HNs co-transfected with the C5 sense oligonucleotide (data not shown), GFP:: $\beta \mathrm{L}$ was diffusely distributed within the cytosol (compare Fig. 4C) and did not accumulate opposite GABAergic terminals. To finally determine whether C5 depletion would also affect endogenous GlyR distribution, we stained GlyRs together with VIAAT in C5 antisense-transfected HNs (Fig. 6D). As compared with the fraction of postsynaptic GlyR clusters in both nontransfected $(33.4 \pm 3.4 \%)$ and C5 sensetransfected HNs $(31.6 \pm 3.4 \%)$, depletion of C5-gephyrins from HNs dramatically facilitated postsynaptic GlyR anchoring opposite GABAergic terminals. In C5 antisense-transfected HNs, $83 \pm$ $3.2 \%$ of the synapses formed by GABA-releasing terminals contained postsynaptic GlyR clusters (Fig. 6E). These results lead to transcripts (bottom panel).
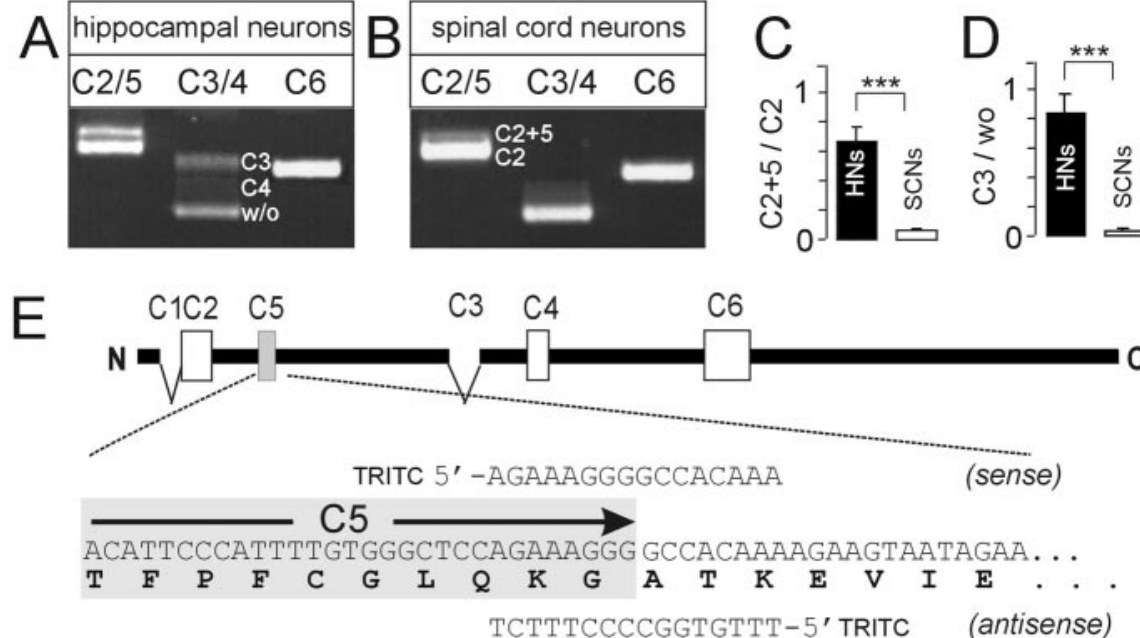

TCTTTCCCCGGTGTTT - 5' TRITC

(antisense)
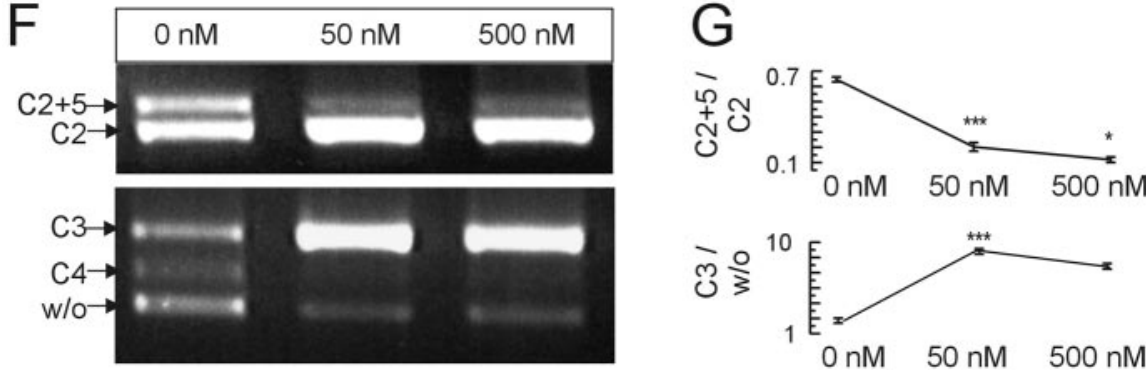

Figure 5. RT-PCR analysis of gephyrin expression in neuronal cultures from HNs (DIV8) and SCNs (DIV12) and C5 antisense oligonucleotide design. $A, B$, Gephyrin isoform expression in $\mathrm{HNs}(A)$ and in $\mathrm{SCNs}(B)$. $C, D$, Quantification of the relative amounts

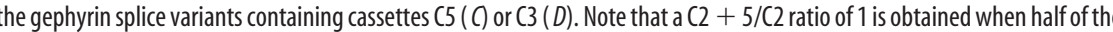
列 transcripts with $\mathrm{C} 2+$ C5 versus transcripts with C2 only (top panel) and concomitant increase in the amount of C3-containing

the conclusion that depletion of C5-containing gephyrins from HNs is sufficient to enable the recruitment of transfected GFP:: $\beta \mathrm{L}$ to gephyrin-containing domains opposite GABAreleasing terminals. This creates presynaptic to postsynaptic mismatches.

\section{Overexpression of C5-gephyrins in SCNs interferes with postsynaptic GlyR stabilization}

If postsynaptic C5-gephyrins provide the basis for a mechanism prohibiting GlyR stabilization, their overexpression should also interfere with the postsynaptic GlyR stabilization in SCNs. To examine this possibility, SCNs were transfected with GeC2,5,6::GFP (Fig. 7A). GeC2,5,6::GFP colocalized with $6.4 \pm$ $3.5,35.7 \pm 7.2$, and $88.0 \pm 6.4 \%$ of glycinergic, mixed, and GABAergic synapses, respectively (Fig. 7B); $83.3 \pm 16.7,28.3 \pm$ 11.1 , and $5.6 \pm 4.6 \%$ of postsynaptic $\mathrm{GeC} 2,5,6:: \mathrm{GFP}$ clusters opposite glycinergic, mixed, and GABAergic synapses, respectively, were immunoreactive for mAb2b antibody (Fig. 7C). The surface area and the average fluorescence intensity of postsynaptic GeC2,5,6::GFP clusters were highest in the case of apposition to GABAergic synapses (Fig. 7D, E) (surface, $1.9 \pm 0.2 \mu \mathrm{m}^{2}$; mean gray, $214.7 \pm 9.8)$. With decreasing GABA content, both the surface area and the mean fluorescence intensity of postsynaptic 

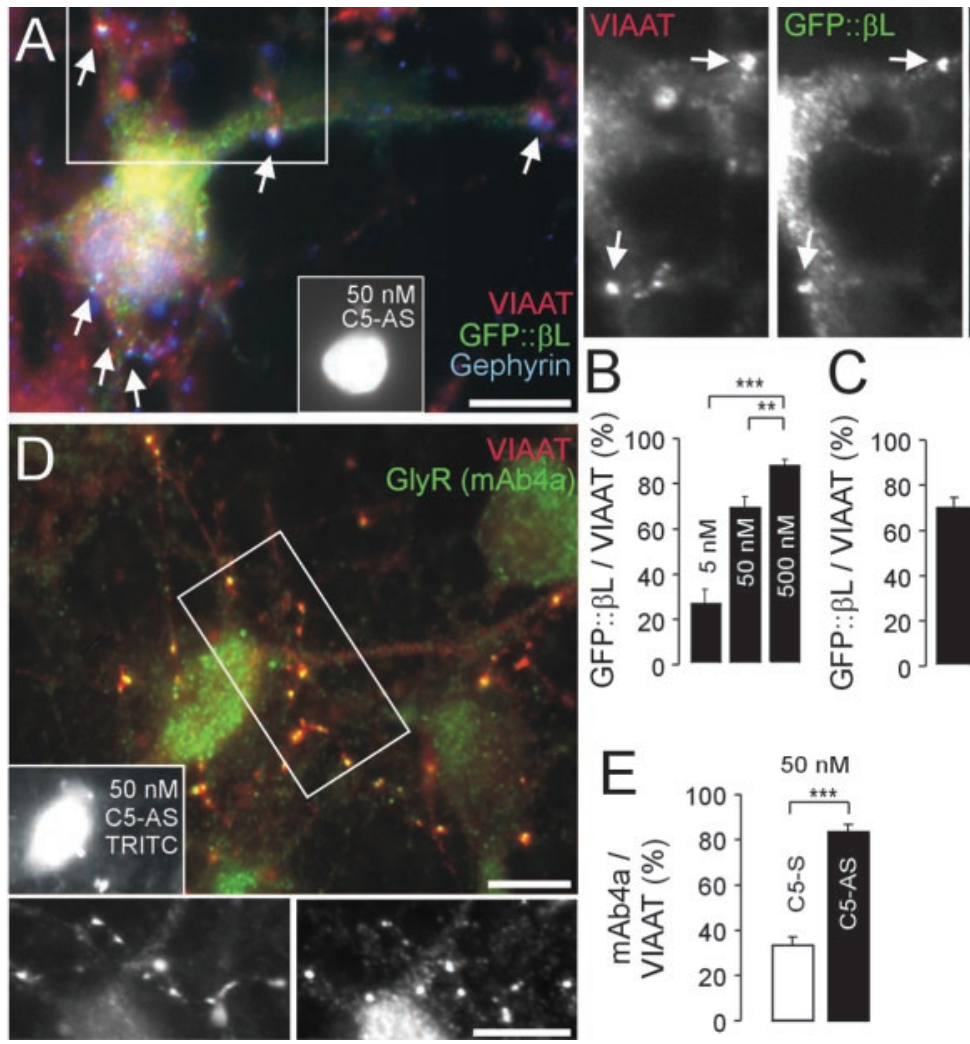

Figure 6. C5-gephyrin depletion in DIV8 HNs allows GFP:: $\beta$ L and endogenous GlyRs to accumulate opposite GABAergic terminals. A, Localization of VIAAT immunoreactivity (red), GFP:: $\beta \mathrm{L}$ (green), and gephyrin (blue) in a $C 5$ antisense co-transfected HN. Inset shows the TRITC signal obtained from the co-transfected $C 5$ antisense oligonucleotide. When $(5$ antisense $(50 \mathrm{~nm})$ is present, aggregates of GFP:: $\beta$ L can be found opposite GABAergic terminals (arrows), in addition to some diffuse GFP:: $\beta L$ signal. Note that postsynaptic GFP:: $\beta \mathrm{L}$ aggregates are colocalized with endogenous gephyrin. The white box in $A$ delineates the region shown at higher magnification. $B$, Fraction of VIAAT-immunoreactive terminals that were colocalized with GFP:: $\beta \mathrm{L}$ is dependent on the concentration of the $(5$ antisense oligonucleotide $(5,50$, and $500 \mathrm{~nm})$. C, Experiment with $50 \mathrm{~nm} C 5$ antisense oligonucleotide. Left ordinate: fraction of VIAAT-immunoreactive spots with colocalized GFP:: $\beta$ L. Right ordinate: fraction of VIAAT and GFP:: $\beta \mathrm{L}$ containing synapses that colocalized with endogenous gephyrin. $D$, Image illustrating the effect of $C 5$ antisense transfection on endogenous GlyR (mAb4a) distribution (inset, TRITC fluorescence). Note the invasion of almost all of the GABAergic synapses with endogenous GlyRs. E, Quantification of the effect of $C 5$ sense and $C 5$ antisense transfection on postsynaptic GlyR accumulation. Scale bars, $10 \mu \mathrm{m}$.

GeC2,5,6::GFP decreased (mixed, $0.6 \pm 0.1 \mu \mathrm{m}^{2}, 127.7 \pm 21.6$; GlyT2, $0.13 \pm 0.05 \mu \mathrm{m}^{2}, 8.7 \pm 0.3$ ) (Fig. $7 D, E$ ).

Together, we have strong evidence to suggest that in SCNs the fraction as well as the amount of C5-gephyrins increase with increasing presynaptic GABA content. The presence of C5gephyrins in the postsynaptic domains interferes with GlyR anchoring, and the degree of interference correlates with the amount of C5-gephyrins present.

\section{Discussion}

SCNs and HNs were transfected to express GFP-tagged gephyrinbinding loops of the GlyR $\beta$ subunit. The loops accumulated at inhibitory gephyrin-containing postsynaptic sites in a transmitterappropriate way. In contrast to SCNs, which form glycinergic, mixed, and purely GABAergic synapses, HNs exclusively make GABAergic contacts. Postsynaptic GFP:: $\beta \mathrm{L}$ aggregates were absent in HNs, and this was associated with high expression levels of C5gephyrins. When transfected with C5 antisense oligonucleotides, HNs erroneously accumulated GFP:: $\beta$ L opposite GABAergic terminals. Accordingly, in SCNs, GeC2,5,6::GFP displayed a preferential postsynaptic accumulation opposite GABAergic synapses. The amount of postsynaptic C5-gephyrins (surface area, fluorescence

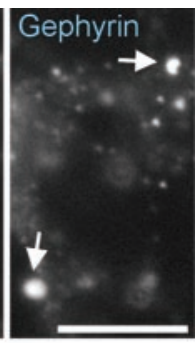

intensity) was positively correlated with the presynaptic GABA content (GlyT2 $<$ mixed $<$ GAD65). We therefore conclude that the amount of C5-gephyrins at inhibitory synapses regulates the access of GlyRs to postsynaptic domains, which contributes to presynaptic to postsynaptic matching (Fig. 8).

The mechanisms of postsynaptic GlyR stabilization have been studied extensively. The currently prevailing concepts of postsynaptic GlyR stabilization imply that GlyR $\beta$ subunits directly bind to gephyrin (Meyer et al., 1995; Kneussel et al., 1999). Our experiments on transfected SCNs showed that the isolated gephyrin-binding loops are competent to accumulate opposite glycine-releasing terminals. This validated the use of the GFP:: $\beta$ L construct as a surrogate for full-length GlyRs in cultured neurons.

In general, a synaptic versus nonsynaptic distribution of GlyRs in neurons receiving (SCNs) or lacking (HNs) glycinergic afferents could be based on various differences, including (1) different intrinsic binding characteristics of the GlyRs and (2) different intrinsic binding characteristics of postsynaptic gephyrins. It is known that transmitter specificity of receptor accumulation may not be absolute. Here we show that in HNs endogenous GlyR clusters can indeed occur opposite GABAergic terminals; however, considering that the GABAergic postsynaptic densities were entirely free of GFP:: $\beta \mathrm{L}$ and endogenous GlyR clusters were visualized by mAb4a, an antibody that recognizes all GlyR subunits, it seems possible that the postsynaptic GlyRs found at a minor fraction of GABAergic presynaptic terminals were composed of homomeric GlyR $\alpha 2$ channels, i.e., they were lacking the GlyR $\beta$ subunit. We have shown previously that homomeric GlyR $\alpha 2$ channels are indeed capable of accumulating at inhibitory postsynaptic sites (Meier et al., 2000b). The function of such mismatched postsynaptic GlyRs in HNs remains to be determined; however, considering their slow onset, relaxation, and desensitization kinetics, as well as the low open probability, it seems unlikely that homomeric $\alpha 2$ GlyR channels contribute much to fast inhibitory synaptic transmission (Mangin et al., 2003).

Our findings support the hypothesis that the amount of C5gephyrins determines the degree of postsynaptic GlyR accumulation, provided these GlyRs contain the gephyrin-binding $\beta$ subunit. Only at purely GABAergic synapses, the amount of C5-gephyrins is sufficient to exclude GlyRs from postsynaptic anchoring. At mixed and purely glycinergic synapses, lower levels of C5-gephyrins will allow for a larger number of postsynaptic GlyRs (Fig. 8A). Accepting that C5-gephyrins keep GABAergic synapses free of heteromeric GlyRs and, vice versa, that gephyrins lacking C5 are required to stabilize heteromeric GlyRs opposite glycinergic terminals, one can conclude that the transmitter specificity of postsynaptic receptor accumulation reflects the presence of an appropriate mixture of gephy- 
rin isoforms. Although it is well documented that alternative splicing produces a large number of gephyrin isoforms (Meier et al., 2000a; Ramming et al., 2000; David-Watine, 2001; Rees et al., 2003), there is still no direct evidence for a similar molecular diversity in the postsynaptic density. Cassette-specific antibodies are not yet available; however, the results obtained in our experiments with transfected GFP-tagged GeC2,5,6 are consistent with the idea that $\mathrm{C} 5$-gephyrins reside predominantly in the postsynaptic protein scaffold of GABAergic synapses where they interfere with GlyR $\beta$ subunit binding. This leads directly to the next question of how C5-gephyrins might achieve the exclusion of heteromeric GlyRs from postsynaptic domains.

It is already known that cassette C5 is inserted into the $\alpha$-helix 4 within the MogAhomologous domain where it may cause a conformational rearrangement that, in turn, influences the orientation of the linker region (Sola et al., 2001). It is therefore possible that C5 insertion into $\alpha$-helix 4 results in some distant action on the C-terminal MoeAhomologous domain, were the GlyR $\beta$ subunit binding domain is located (Rees et al., 2003). This could lead to a masking of the GlyR $\beta$ subunit binding site. The ability to mask the GlyR-binding domain in the C-terminal part may be an exclusive property of C5-containing gephyrins (Fig. $8 \mathrm{~B}$ ).

It is conceivable that $\mathrm{C} 5$ insertion into $\alpha$-helix 4 can provoke additional structural changes within the highly conserved Gly-GlyThr-Gly-motif, which may affect the trimer interface within the Gephyrin-G (MogA homologous) domain (Schwarz et al., 2001). Conformational rearrangements at the trimer interface may exert remote effects on adjacent gephyrins that eventually may distort the postsynaptic gephyrin lattice in the vicinity of C5-gephyrins (Fig. $8 C)$. This hypothesis is supported by the observation that the presence of cassette C5 altered the aggregation behavior of gephyrin. When excessively overexpressed in COS cells or neurons, C5gephyrins tended to form filamentous, spike-like aggregates, whereas gephyrins lacking C5 aggregated into blob-like structures (Meier et al., 2000a).

This notion leads to the question of how the postsynaptic neuron is instructed to target the appropriate gephyrin isoforms to inhibitory postsynaptic sites. Mismatches in the apposition of presynaptic and postsynaptic components were created, for instance, in neurons manipulated to lack one or several synaptic afferents. In cultures of solitary motoneurons, gephyrin accumulated opposite the remaining cholinergic autaptic terminals (Levi et al., 1999). In pyramidal cells deprived of GABAergic input, $\mathrm{GABA}_{\mathrm{A}}$ receptors accumulated opposite glutamatergic terminals (Rao et al., 2000). Although the nature of the signal still remains to be determined, these data strongly suggest that the instructive signal necessary to obtain the appropriate postsynaptic gephyrin heterogeneity is provided by the presynaptic terminal.

Because invasion of GlyRs into GABAergic postsynaptic sites is not a priori excluded but reflects the level of C5-gephyrins present in the postsynaptic domains, one can conclude that this mechanism gives GABAergic synapses an additional degree of freedom to ensure a functionally adequate level of inhibition, e.g., when GABA release for some reasons drops to very low level. The
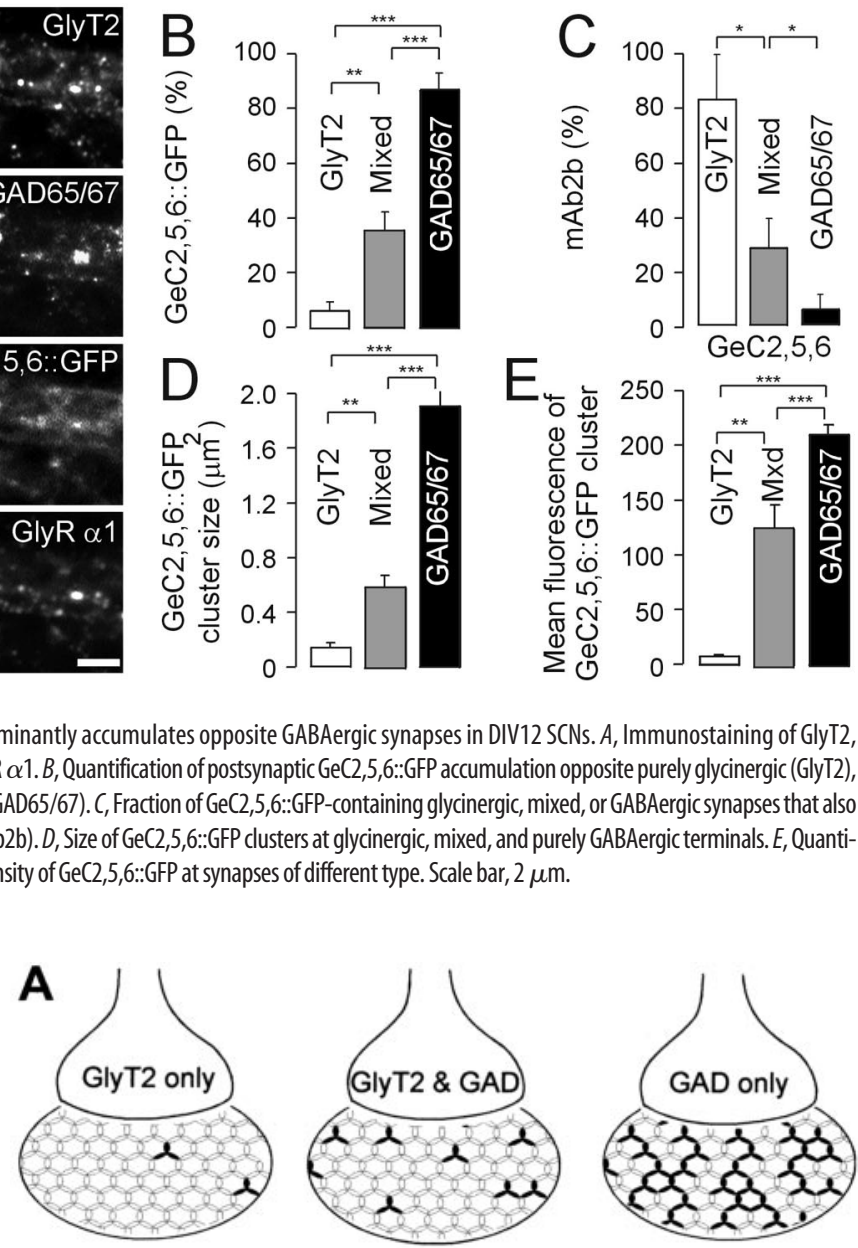
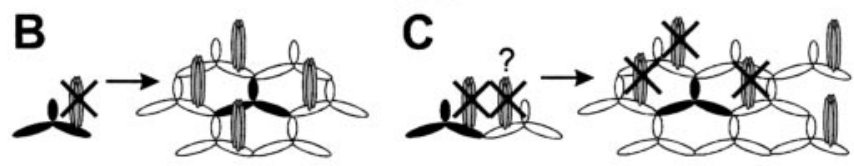

\section{d C5-containing gephyrins} \& Gephyrins lacking C5

$\beta$ subunit-containing GlyR channel

Figure 8. Scheme to illustrate the proposed mechanism for C5-gephyrin-dependent control of GlyR anchoring. A, Postsynaptic lattice containing a variable amount of $(5$-gephyrin trimers (black). The highest amount is present in purely GABAergic synapses. The suppression of GlyR accumulation may either be limited to the 5 -gephyrin sites $(B)$ or additionally involve the area occupied by neighboring $C 5$-lacking gephyrins, provided the latter are also under the influence of C5-gephyrins.

conditional GlyR stabilization at postsynaptic GABAergic densities thus may represent an interesting new form of plasticity. It has been shown by in vivo microdialysis that the concentration of glycine increases during and after induction of epilepsy (Shen and Lai, 2002). Provided that extracellular glycine can be taken up by presynaptic terminals, which may indeed occur even in the absence of GlyT2 (Gomeza et al., 2003), additional synaptic GlyR clusters could secure an appropriate level of synaptic inhibition.

\section{References}

Beaulieu C, Campistron G, Crevier C (1994) Quantitative aspects of the GABA circuitry in the primary visual cortex of the adult rat. J Comp Neurol 339:559-572.

Brewer GJ, Cotman CW (1989) Survival and growth of hippocampal neurons in defined medium at low density: advantages of a sandwich culture technique or low oxygen. Brain Res 494:65-74. 
Butler MH, Hayashi A, Ohkoshi N, Villmann C, Becker CM, Feng G, De Camilli P, Solimena M (2000) Autoimmunity to gephyrin in Stiff-Man syndrome. Neuron 26:307-312.

David-Watine B (2001) The human gephyrin (GPHN) gene: structure, chromosome localization and expression in non-neuronal cells. Gene 271:239-245.

Dumoulin A, Rostaing P, Bedet C, Levi S, Isambert MF, Henry JP, Triller A, Gasnier B (1999) Presence of the vesicular inhibitory amino acid transporter in GABAergic and glycinergic synaptic terminal boutons. J Cell Sci 112:811-823.

Dumoulin A, Levi S, Riveau B, Gasnier B, Triller A (2000) Formation of mixed glycine and GABAergic synapses in cultured spinal cord neurons. Eur J Neurosci 12:3883-3892.

Gasnier B (2000) The loading of neurotransmitters into synaptic vesicles. Biochimie 82:327-337.

Gomeza J, Ohno K, Hulsmann S, Armsen W, Eulenburg V, Richter DW, Laube B, Betz H (2003) Deletion of the mouse glycine transporter 2 results in a hyperekplexia phenotype and postnatal lethality. Neuron 40:797-806.

Gottlieb DI, Chang YC, Schwob JE (1986) Monoclonal antibodies to glutamic acid decarboxylase. Proc Natl Acad Sci USA 83:8808-8812.

Ito S, Cherubini E (1991) Strychnine-sensitive glycine responses of neonatal rat hippocampal neurones. J Physiol (Lond) 440:67-83.

Jonas P, Bischofberger J, Sandkuhler J (1998) Corelease of two fast neurotransmitters at a central synapse. Science 281:419-424.

Kins S, Kuhse J, Laube B, Betz H, Kirsch J (1999) Incorporation of a gephyrin-binding motif targets NMDA receptors to gephyrin-rich domains in HEK 293 cells. Eur J Neurosci 11:740-744.

Kneussel M, Hermann A, Kirsch J, Betz H (1999) Hydrophobic interactions mediate binding of the glycine receptor beta-subunit to gephyrin. J Neurochem 72:1323-1326.

Levi S, Chesnoy-Marchais D, Sieghart W, Triller A (1999) Synaptic control of glycine and $\mathrm{GABA}(\mathrm{A})$ receptors and gephyrin expression in cultured motoneurons. J Neurosci 19:7434-7449.

Luhmann HJ, Prince DA (1991) Postnatal maturation of the GABAergic system in rat neocortex. J Neurophysiol 65:247-263.

Mangin JM, Baloul M, Prado DC, Rogister B, Rigo JM, Legendre P (2003) Kinetics properties of the $\{$ alpha\} 2 homo-oligomeric glycine receptor impairs a proper synaptic functioning. J Physiol (Lond) 553:369-386.

McIntire SL, Reimer RJ, Schuske K, Edwards RH, Jorgensen EM (1997) Identification and characterization of the vesicular GABA transporter. Nature 389:870-876.

Meier J, De Chaldee M, Triller A, Vannier C (2000a) Functional heterogeneity of gephyrins. Mol Cell Neurosci 16:566-577.

Meier J, Meunier-Durmort C, Forest C, Triller A, Vannier C (2000b) Formation of glycine receptor clusters and their accumulation at synapses. J Cell Sci 113:2783-2795.

Meier J, Vannier C, Serge A, Triller A, Choquet D (2001) Fast and reversible trapping of surface glycine receptors by gephyrin. Nat Neurosci 4:253-260.

Meier J, Jüttner R, Kirischuk S, Grantyn R (2002) Synaptic anchoring of glycine receptors in developing collicular neurons under control of metabotropic glutamate receptor activity. Mol Cell Neurosci 21:324-340.

Meier J, Akyeli J, Kirischuk S, Grantyn R (2003) GABAA receptor activity and $\mathrm{PKC}$ control inhibitory synaptogenesis in CNS tissue slices. Mol Cell Neurosci 23:600-613.

Meyer G, Kirsch J, Betz H, Langosch D (1995) Identification of a gephyrin binding motif on the glycine receptor beta subunit. Neuron 15:563-572.

Mori M, Gahwiler BH, Gerber U (2002) $\beta$-Alanine and taurine as endogenous agonists at glycine receptors in rat hippocampus in vitro. J Physiol (Lond) 539:191-200.
Nicoll RA, Malenka RC (1998) A tale of two transmitters. Science 281:360-361

Pfeiffer F, Simler R, Grenningloh G, Betz H (1984) Monoclonal antibodies and peptide mapping reveal structural similarities between the subunits of the glycine receptor of rat spinal cord. Proc Natl Acad Sci USA 81:7224-7227.

Prior P, Schmitt B, Grenningloh G, Pribilla I, Multhaup G, Beyreuther K, Maulet Y, Werner P, Langosch D, Kirsch J (1992) Primary structure and alternative splice variants of gephyrin, a putative glycine receptor-tubulin linker protein. Neuron 8:1161-1170.

Radian R, Ottersen OP, Storm-Mathisen J, Castel M, Kanner BI (1990) Immunocytochemical localization of the GABA transporter in rat brain. J Neurosci 10:1319-1330.

Ramming M, Kins S, Werner N, Hermann A, Betz H, Kirsch J (2000) Diversity and phylogeny of gephyrin: tissue-specific splice variants, gene structure, and sequence similarities to molybdenum cofactor-synthesizing and cytoskeleton-associated proteins. Proc Natl Acad Sci USA 97:10266-10271.

Rao A, Kim E, Sheng M, Craig AM (1998) Heterogeneity in the molecular composition of excitatory postsynaptic sites during development of hippocampal neurons in culture. J Neurosci 18:1217-1229.

Rao A, Cha EM, Craig AM (2000) Mismatched appositions of presynaptic and postsynaptic components in isolated hippocampal neurons. J Neurosci 20:8344-8353.

Rees MI, Harvey K, Ward H, White JH, Evans LI, Duguid IC, Hsu CC Coleman SL, Miller J, Baer K, Waldvogel HJ, Gibbon F, Smart TG, Owen MJ, Harvey RJ, Snell RG (2003) Isoform heterogeneity of the human gephyrin gene (GPHN), binding domains to the glycine receptor and mutation analysis in hyperekplexia. J Biol Chem 278:24688-24696.

Reiss J, Johnson JL (2003) Mutations in the molybdenum cofactor biosynthetic genes MOCS1, MOCS2, and GEPH. Hum Mutat 21:569-576.

Reiss J, Gross-Hardt S, Christensen E, Schmidt P, Mendel RR, Schwarz G (2001) A mutation in the gene for the neurotransmitter receptorclustering protein gephyrin causes a novel form of molybdenum cofactor deficiency. Am J Hum Genet 68:208-213.

Sagné C, El Mestikawy S, Isambert MF, Hamon M, Henry JP, Giros B, Gasnier B (1997) Cloning of a functional vesicular GABA and glycine transporter by screening of genome databases. FEBS Lett 417:177-183.

Schwarz G, Schrader N, Mendel RR, Hecht HJ, Schindelin H (2001) Crystal structures of human gephyrin and plant cnxl g domains: comparative analysis and functional implications. J Mol Biol 312:405-418.

Shen EY, Lai YJ (2002) In vivo microdialysis study of excitatory and inhibitory amino acid levels in the hippocampus following penicillin-induced seizures in mature rats. Acta Paediatr Taiwan 43:313-318.

Soghomonian JJ, Martin DL (1998) Two isoforms of glutamate decarboxylase: why? Trends Pharmacol Sci 19:500-505.

Sola M, Kneussel M, Heck IS, Betz H, Weissenhorn W (2001) X-ray crystal structure of the trimeric N-terminal domain of gephyrin. J Biol Chem 276:25294-25301.

Thio LL, Shanmugam A, Isenberg K, Yamada K (2003) Benzodiazepines block $\alpha 2$-containing inhibitory glycine receptors in embryonic mouse hippocampal neurons. J Neurophysiol 90:89-99.

Xiang S, Nichols J, Rajagopalan KV, Schindelin H (2001) The crystal structure of Escherichia coli MoeA and its relationship to the multifunctiona protein gephyrin. Structure 9:299-310.

Zafra F, Aragon C, Olivares L, Danbolt NC, Gimenez C, Storm-Mathisen J (1995a) Glycine transporters are differentially expressed among CNS cells. J Neurosci 15:3952-3969.

Zafra F, Gomeza J, Olivares L, Aragon C, Gimenez C (1995b) Regional distribution and developmental variation of the glycine transporters GLYT1 and GLYT2 in the rat CNS. Eur J Neurosci 7:1342-1352. 